\title{
Sodium imaging of the heart at 7T: design, evaluation and application of a four-channel transmit/receive surface coil array
}

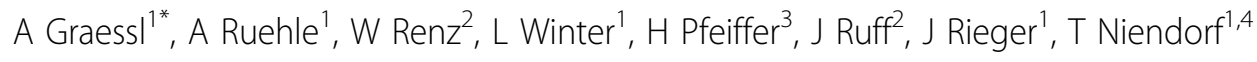 \\ From 16th Annual SCMR Scientific Sessions \\ San Francisco, CA, USA. 31 January - 3 February 2013
}

\section{Background}

Insight of physiological processes and cellular metabolism makes 23Na-MRI conceptually appealing as non-invasive imaging discipline. Several studies report the applicability of 23Na-MRI for the detection and assessment of acute and chronic heart disease due to increased sodium concentration after myocardial infarctions. Bi-exponential decay of the signal and a low SNR compared to $1 \mathrm{H}-\mathrm{MRI}$ makes 23Na-MRI unattractive for clinical use. With a high SNR and fast imaging technologies ultrahigh field MRI brings $23 \mathrm{Na}$-MRI back into focus, asking for dedicated radiofrequency (RF) technology.

\section{Methods}

The proposed four channel transceiver RF-coil tuned to $78.6 \mathrm{MHz}$ consists of an anterior and a posterior section (Fig 1), each containing two $210 \times 140 \mathrm{~mm}$ rectangular loop elements connected with a shared conductor. EMF and SAR simulations were performed using CST Studio Suite 2011 (CST AG, Darmstadt, Germany) together with the voxel model Duke from the Virtual family (ITIS Foundation, Zurich, Switzerland). In-vivo studies were performed in healthy volunteers using a $7 \mathrm{~T}$ whole body MRI system (Magnetom, Siemens, Erlangen, Germany). The volunteers were positioned prone to reduce artifacts due to respiratory motion. $23 \mathrm{Na}$ localizer imaging was performed with untriggered gradient echo (2D FLASH) imaging (FOV $320 \times 380, T E=2,28 \mathrm{~ms}, \mathrm{TR}=5,6 \mathrm{~ms}$; $\mathrm{TA}=1,32 \mathrm{~min}$; matrix size $5 \times 5 \times 40 \mathrm{~mm}$, averages 256 ).
Short axis images were acquired with a cardiac triggered gradient echo (2D FLASH) sequence (FOV 282x379, $\mathrm{TE}=2,24 \mathrm{~ms}$, $\mathrm{TR}=200 \mathrm{~ms}$; $\mathrm{TA}=11,23 \mathrm{~min}$; matrix size $5,4 \times 5,4 \times 40 \mathrm{~mm}$, averages 400 ) of the heart. For triggering an acoustic cardiac gating device (easyACT, MRI.Tools $\mathrm{GmbH}$, Germany) was used.

\section{Results}

The reflection coefficient of each element was measured to be better than $-18 \mathrm{~dB}$, transmission coefficients were found to be below $-16 \mathrm{~dB}$. The SAR values fell well within the limits provided by the IEC 606012-33 for input power of $10 \mathrm{~W}$ in normal mode. In vivo studies yielded a rather uniform signal intensity across the heart leading to adequate image quality. An intense signal caused by the high $23 \mathrm{Na}$-concentration of the ribs was observed. The cardiac triggered acquisitions showed a SNR of 38 in the blood-pool and 24 in the septum resulting in a blood/myocardium contrast of 3,3 .

\section{Conclusions}

Our results demonstrate that 23Na-MRI of the heart is feasible at 7.0 $\mathrm{T}$. The proposed RF coil design yielded adequate image quality within clinically acceptable scan times for free breathing, cardiac triggered acquisitions. Using an even larger number of TX/RX channels would help to further boost SNR and spatial resolution together with scan time shortening. 


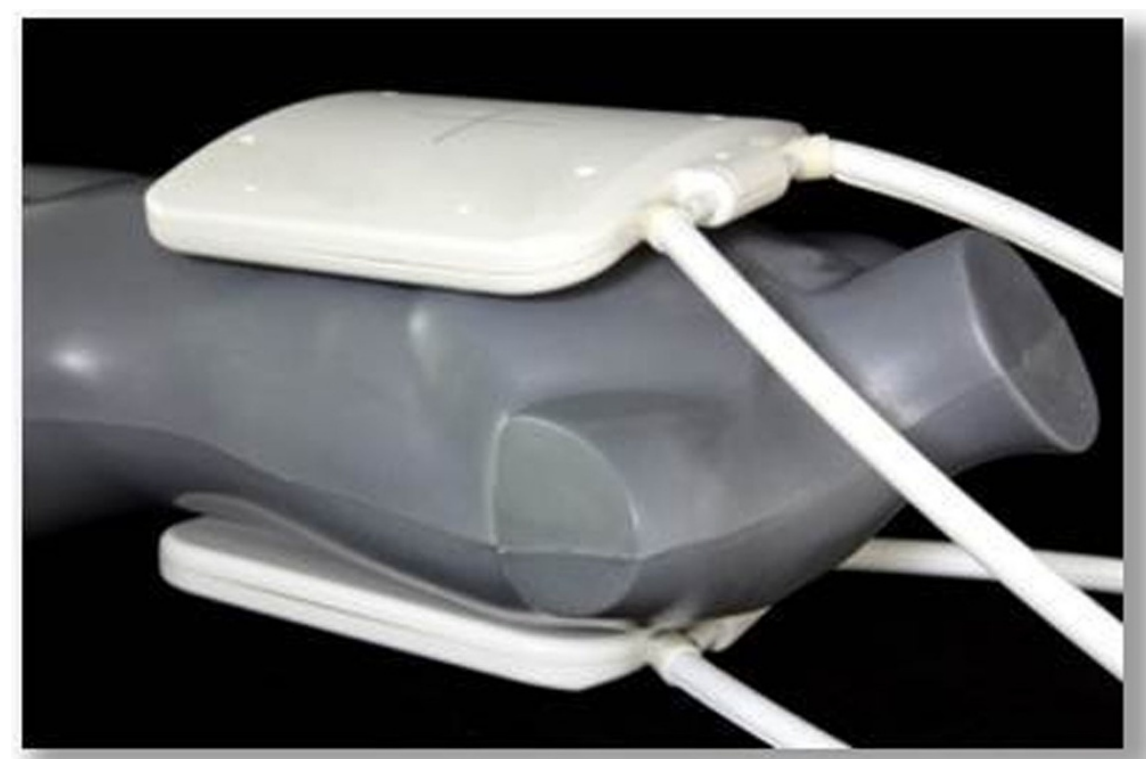

Figure 1 The coil housed in two modestly curved lightweight formers to conform to an average chest and back, shown on a mannequin.

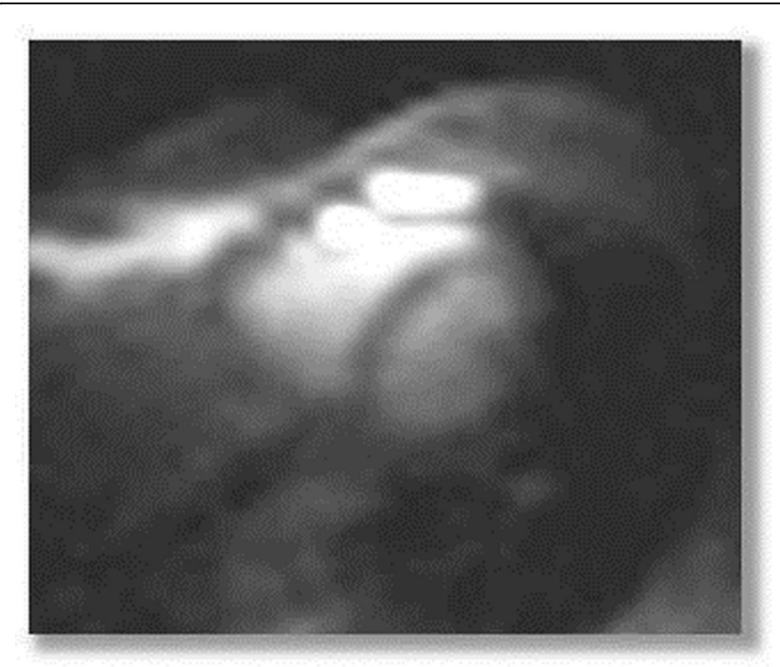

Figure $223 \mathrm{Na}$ short axis view of the heart acquired with an cardiac triggered 2D FLASH sequence.

\section{Author details}

${ }^{1}$ Max-Delbrueck-Center for Molecular Medicine, Berlin, Germany. ${ }^{2}$ Siemens Healthcare, Erlangen, Germany. ${ }^{3}$ Physikalisch-Technische Bundesanstalt (PTB), Berlin, Germany. ${ }^{4}$ Experimental and Clinical Research Center (ECRC), Charité Campus Buch, Humboldt-University, Berlin, Germany.

Published: 30 January 2013

\section{doi:10.1186/1532-429X-15-S1-W14}

Cite this article as: Graessl et al: Sodium imaging of the heart at $7 \mathrm{~T}$ : design, evaluation and application of a four-channel transmit/receive surface coil array. Journal of Cardiovascular Magnetic Resonance 201315 (Suppl 1):W14.

\section{Submit your next manuscript to BioMed Central} and take full advantage of:

- Convenient online submission

- Thorough peer review

- No space constraints or color figure charges

- Immediate publication on acceptance

- Inclusion in PubMed, CAS, Scopus and Google Scholar

- Research which is freely available for redistribution 